\title{
Correction to: Effects of Blood Flow Restriction Training on Muscular Strength and Hypertrophy in Older Individuals: A Systematic Review and Meta-Analysis
}

\author{
Christoph Centner $^{1}\left[\right.$ [ $\cdot$ Patrick Wiegel ${ }^{1,2}$ (]) $\cdot$ Albert Gollhofer $^{1} \cdot$ Daniel König $^{1}$
}

Published online: 9 November 2018

(c) Springer Nature Switzerland AG 2018

Correction to: Sports Medicine (2018)

https://doi.org/10.1007/s40279-018-0994-1

Section 3.2: Fig. 2 and Fig. 3 were transposed.

Figure 2 should be:

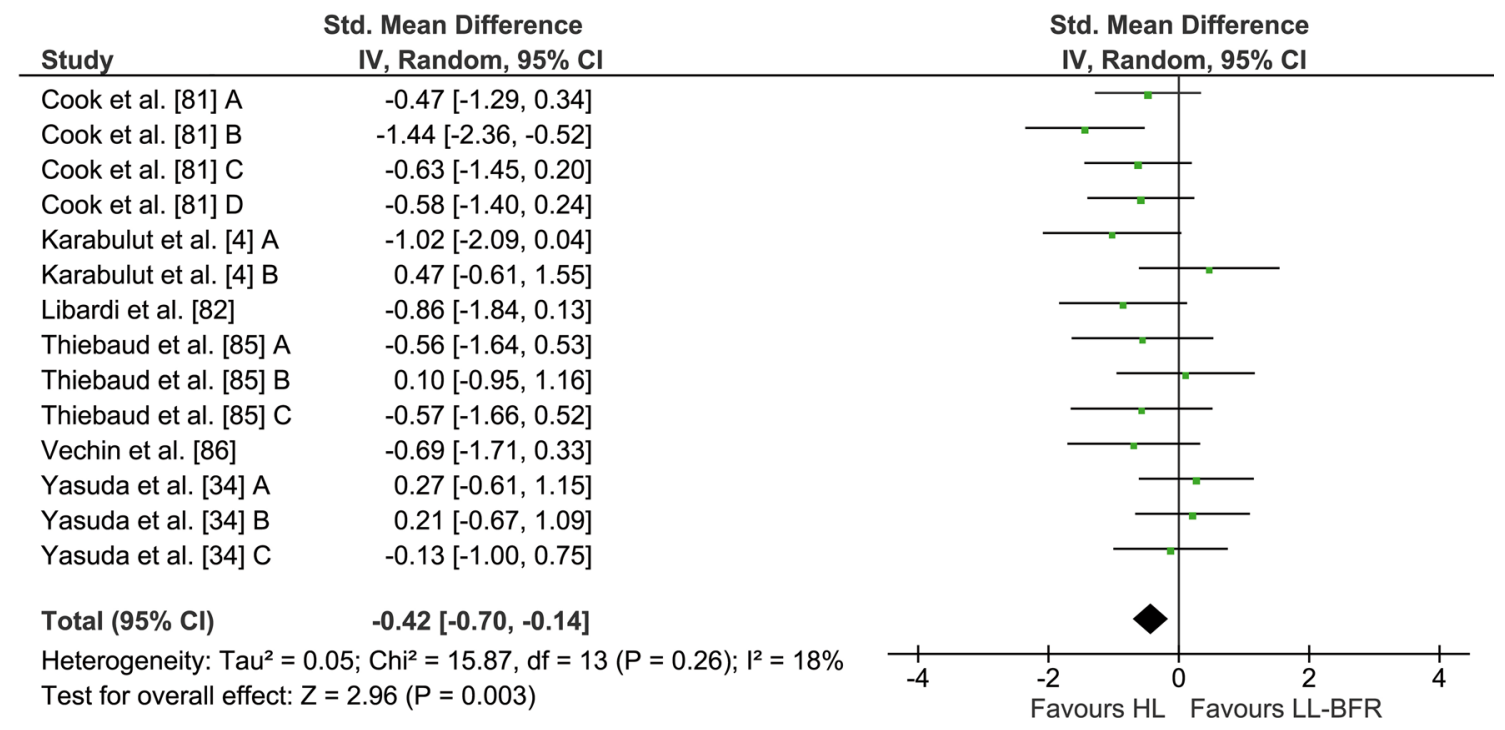

Fig. 2 Forest plot demonstrating the effects of LL-BFR versus HL training on muscular strength. Different letters for the same study represent different muscular strength assessment methods. $C I$ confidence interval, $H L$ high-load, $I V$ inverse variance, $L L-B F R$ low-load blood flow restriction, Random random effects model

The original article can be found online at https://doi.org/10.1007/ s40279-018-0994-1.

Christoph Centner

christoph.centner@sport.uni-freiburg.de

1 Department of Sport and Sport Science, University of Freiburg, Freiburg, Germany

2 Bernstein Center Freiburg, University of Freiburg, Freiburg, Germany 
Figure 3 should be:

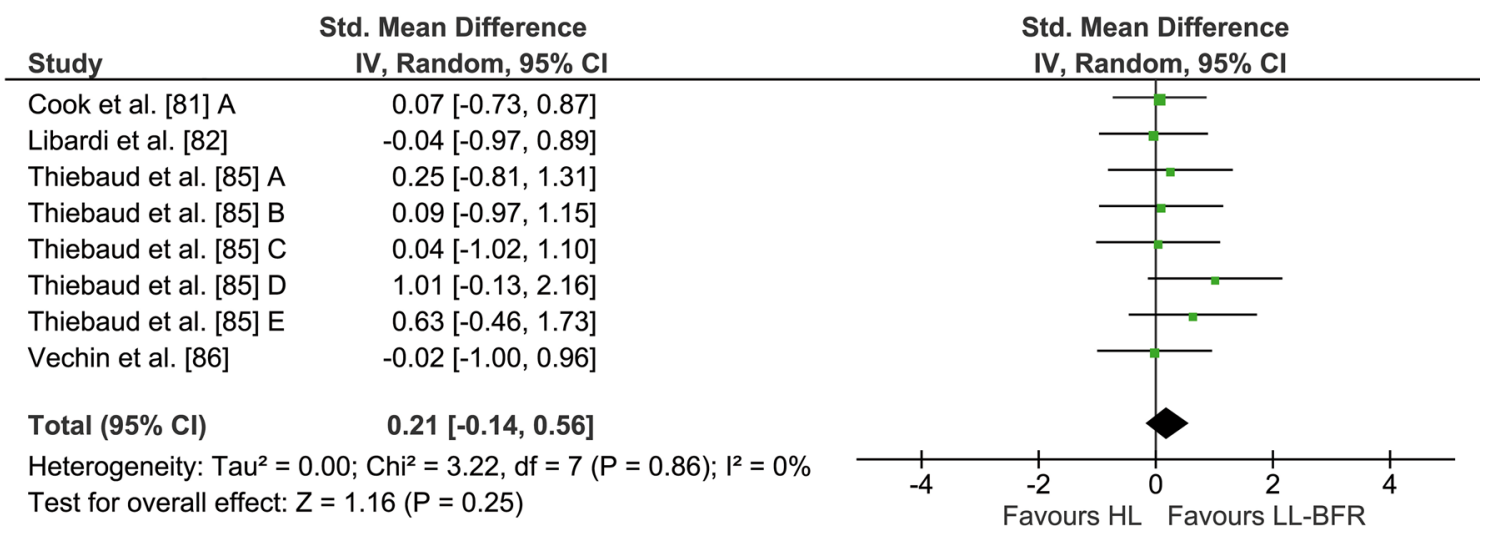

Fig. 3 Forest plot demonstrating the effects of LL-BFR versus HL training on muscle mass. Different letters for the same study represent different assessment methods for muscle mass. $C I$ confidence inter- val, $H L$ high-load, $I V$ inverse variance, $L L-B F R$ low-load blood flow restriction, Random random effects model

Section 3.4: Fig. 5 and Fig. 6 were transposed.

Figure 5 should be:

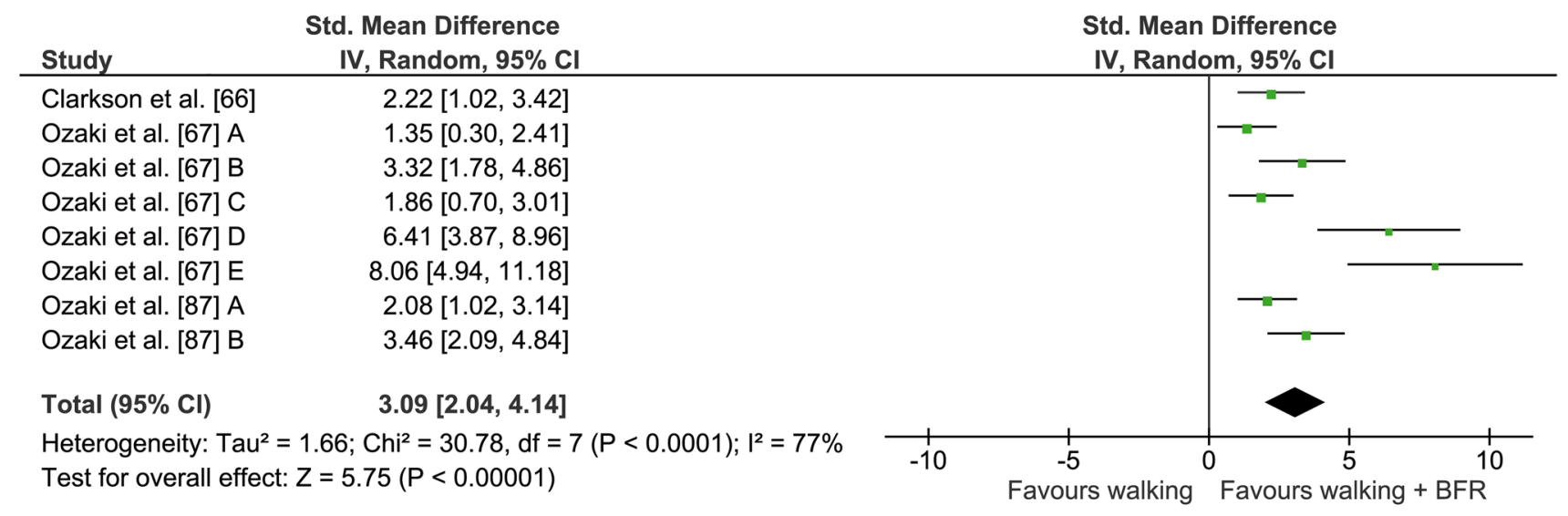

Fig. 5 Forest plot demonstrating the effects of walking + BFR versus normal walking on muscular strength. Different letters for the same study represent different muscular strength assessment methods. BFR blood flow restriction, $C I$ confidence interval, $I V$ inverse variance, Random random effects model 
Figure 6 should be:

Std. Mean Difference

Study

Ozaki et al. [67] A

Ozaki et al. [67] B

Ozaki et al. [67] C

Ozaki et al. [67] D

Ozaki et al. [87] A

Total $(95 \% \mathrm{Cl})$

Heterogeneity: $\mathrm{Tau}^{2}=0.00 ; \mathrm{Chi}^{2}=1.33, \mathrm{df}=4(\mathrm{P}=0.86) ; \mathrm{I}^{2}=0 \%$

Test for overall effect: $Z=7.11(P<0.00001)$

Fig. 6 Forest plot demonstrating the effects of walking + BFR versus normal walking on muscle mass. Different letters for the same study represent different muscle mass assessment methods. BFR blood flow

$1.49[0.41,2.58]$

$2.40[1.11,3.68]$

$1.92[0.75,3.09]$

$1.59[0.49,2.69]$

$1.85[0.83,2.86]$

$1.82[1.32,2.32]$
Std. Mean Difference

IV, Random, $95 \% \mathrm{CI}$

restriction, $C I$ confidence interval, $I V$ inverse variance, Random random effects model

The original article has been corrected. 